\title{
Reachability computations for constrained discrete-time systems with state- and input-dependent disturbances
}

\author{
Sas̆a V. Raković, Eric C. Kerrigan ${ }^{\dagger \ddagger}$ and David Q. Mayne* \\ Technical Report EEE/C\&P/SVR/8-a/2003, Imperial College London \\ August 15, 2003
}

\begin{abstract}
This paper presents new results that allow one to compute the set of states which can be robustly steered in a finite number of steps, via state feedback control, to a given target set. The assumptions that are made in this paper are that the system is discrete-time, nonlinear and time-invariant and subject to mixed constraints on the state and input. A persistent disturbance, dependent on the current state and input, acts on the system. Existing results are not able to address state- and input-dependent disturbances and the results in this paper are therefore a generalization of previously-published results. The application of the results to the computation of the maximal robustly controlled invariant set is also briefly discussed. Specific results, which allow one to perform the set computations using polyhedral algebra, linear programming and computational geometry software, are presented for linear and piecewise affine systems with additive state disturbances. Some simple examples are given which show that, even if all the relevant sets are convex and the system is linear, convexity of the robustly controllable sets cannot be guaranteed.

Keywords: Constrained control, robust control, nonlinear systems, piecewise affine systems, set invariance, controllability, uncertain systems.
\end{abstract}

\section{Introduction}

The problems of controllability to a target set and computation of robustly controlled invariant sets for systems subject to constraints and persistent, unmeasured disturbances have been the subject of study for many authors $[2,3,5,8,9,11-13,17]$. Though many papers have fairly general results that can be applied to a large class of nonlinear discrete-time systems, most authors assume that the disturbance is not dependent on the state and input. The only paper which appears to address state-dependent disturbances directly is [8]. In [11] a general framework is introduced for systems with mixed state and input constraints subject to stateand input-dependent disturbances, but the only specific results, which allow one to compute

\footnotetext{
${ }^{*}$ Department of Electrical and Electronic Engineering, Imperial College, London SW7 2BT, United Kingdom. Tel: +44-(0)20-7594-6295/87/81. Fax: +44-(0)20-7594-6282, E-mail: sasa.rakovic@imperial.ac.uk and d.mayne@imperial.ac.uk

${ }^{\dagger}$ Department of Engineering, University of Cambridge, Trumpington Street, Cambridge CB2 1PZ, United Kingdom. Tel: +44-(0)1223-332600. Fax: +44-(0)1223-332662. Email: erickerrigan@ieee.org

${ }^{\ddagger}$ Royal Academy of Engineering Post-doctoral Research Fellow.
} 
the set of states from which the system can be controlled to a target set, are given for disturbances which are independent of the state and input. This paper therefore extends the results of $[8,11,12]$ to the case where the disturbance is dependent on the state and input. Furthermore, results are given for linear and piecewise affine systems which allow the use of polyhedral algebra, linear programming and computational geometry software to perform the set computations.

The need for a framework which can deal with state- and input-dependent disturbances was briefly motivated in [11]. Disturbances that are dependent on the state and/or input frequently arise in practice when trying to model systems with physical constraints. For example, consider the nonlinear (piecewise affine) system

$$
x^{+}=A x+B \operatorname{sat}_{u}\left(u+E_{u} w\right)+E_{x} w
$$

which is subject to a bounded disturbance $w \in \mathcal{W}$. The function $\operatorname{sat}_{u}(\cdot)$ models physical saturation limits on the input. Assuming that these saturation limits are symmetric and have unit magnitude, an equivalent way of modelling (1) is to treat it as linear system with input-dependent disturbances, i.e. letting

$$
x^{+}=A x+B u+B E_{u} w+E_{x} w,
$$

where the control is constrained to

$$
\mathcal{U}:=\left\{u \mid\|u\|_{\infty} \leq 1\right\}
$$

and the input-dependent disturbance $w \in \mathcal{W}(u)$ satisfies

$$
\mathcal{W}(u):=\left\{w \mid\left\|u+E_{u} w\right\|_{\infty} \leq 1 \text { and } w \in \mathcal{W}\right\}
$$

Another common reason why state- and input-dependent disturbances arise in practice is when it is known that the uncertainty of a model is greater in certain regions of the state-input space than in other regions. For example, when a nonlinear model is linearized, the uncertainty gets larger the further one gets from the point of linearization. This uncertainty can be modelled as a state- and input-dependent disturbance, where the size of the disturbance decreases the closer one gets to the point of linearization. A state- and input-dependent disturbance model will therefore allow one to obtain less conservative results than if one were to assume that the disturbance is independent of the state and input.

Another example when one can model uncertainty as a state- and input-dependent disturbance is when there is parametric uncertainty present in the model. For example, if there is uncertainty in the pair $(A, B)$ in $(2)$, then one can think of the uncertainty as an additional state- and input-dependent disturbance. The reader is referred to [4] to see how reachability computations can be carried out for this specific class of uncertainty when the system is linear. The results in this paper can, with some effort, be used to extend the results in [4] to the class of piecewise affine systems with parametric uncertainty.

This paper is organized as follows. Section 2 presents the main results of this paper and Section 3 briefly discusses how the results in Section 2 can be used to iteratively compute the set of states that can be steered to a target set in a finite number of steps, as well as how one could compute the maximal robustly controlled invariant set. In order to validate 
the results presented in this paper, Section 4 presents a few simple numerical examples. The main contributions of this paper are summarized in Section 5. Appendix A contains some results regarding continuity of set-valued maps and Appendix B gives some new results that allow one to compute the set difference of (possibly non-convex) polygons.

Notation and definitions: The set difference of $A \subset \mathbf{R}^{n}$ and $B \subset \mathbf{R}^{n}$ is $A \backslash B:=$ $\{x \in A \mid x \notin B\}=A \cap B^{\mathrm{c}}$, where $B^{\mathrm{c}}$ is the complement of $B .2^{A}$ is the set of subsets of $A$. Given a set $\Omega \subset C \times D$, the projection of $\Omega$ onto $C$ is defined as $\operatorname{Proj}_{C}(\Omega):=$ $\{c \in C \mid \exists d \in D$ such that $(c, d) \in \Omega\}$. convh $\left\{v_{1}, \ldots, v_{n}\right\}$ is the convex hull of the set of points $\left\{v_{1}, \ldots, v_{n}\right\}$. A polyhedron is the (convex) intersection of a finite number of open and/or closed half-spaces and a polygon is the (possibly non-convex) union of a finite number of polyhedra.

\section{The One-step Robustly Controllable set}

Section 2.1 gives the main results of the paper which are then specialized in Section 2.2 for the case when the disturbance is dependent only on the state or input or when the system does not have a control input. Section 2.3 shows that the set of states robustly controllable to the target set is a polygon if the system is linear/affine or piecewise affine, the target set is a polygon and all relevant constraint sets are polygons.

\subsection{General Case}

Consider the time-invariant discrete-time system

$$
x^{+}=f(x, u, w),
$$

where $x$ is the current state (assumed to be measured), $x^{+}$is the successor state, $u$ is the input, and $w$ is an unmeasured, persistent disturbance that is dependent on the current state and input:

$$
w \in \mathcal{W}(x, u) \subset W
$$

where $W=\mathbf{R}^{p}$ denotes the disturbance space. The state and input are required to satisfy the constraints

$$
(x, u) \in \mathcal{Y} \subset X \times U,
$$

where $X=\mathbf{R}^{n}$ is the state space and $U=\mathbf{R}^{m}$ is the input space. The constraint $(x, u) \in \mathcal{Y}$ defines the state-dependent set of admissible inputs

$$
\mathcal{U}(x):=\{u \mid(x, u) \in \mathcal{Y}\}
$$

as well as the set of admissible states

$$
\mathcal{X}:=\{x \mid \exists u \text { such that }(x, u) \in \mathcal{Y}\}=\{x \mid \mathcal{U}(x) \neq \emptyset\} .
$$

In order to have a well-defined problem, we assume the following:

A1. For all $(x, u) \in \mathcal{Y}, \mathcal{W}(x, u) \neq \emptyset$ and $\mathcal{W}(\cdot)$ is bounded on bounded sets.

Given a set $\Omega \subseteq \mathcal{X}$, this section shows how the one-step robustly controllable set (the set of states $\operatorname{Pre}(\Omega)$ for which there exists an admissible input such that, for all allowable disturbances, the successor state is in $\Omega$ may be computed. The set $\operatorname{Pre}(\Omega)$ is defined by

$$
\operatorname{Pre}(\Omega):=\{x \mid \exists u \in \mathcal{U}(x) \text { such that } f(x, u, w) \in \Omega \text { for all } w \in \mathcal{W}(x, u)\} .
$$


Remark 1. If the constraints on the state and input are independent, i.e. $\mathcal{Y}=\mathcal{X} \times \mathcal{U}$, then

$$
\operatorname{Pre}(\Omega)=\{x \in \mathcal{X} \mid \exists u \in \mathcal{U} \text { such that } f(x, u, \mathcal{W}(x, u)) \subseteq \Omega\} .
$$

Theorem 1 (Main result). Let

$$
\Sigma:=\{(x, u) \in \mathcal{Y} \mid f(x, u, w) \in \Omega \text { for all } w \in \mathcal{W}(x, u)\}
$$

and

$$
\Pi:=\{(x, u, w) \mid(x, u) \in \mathcal{Y} \text { and } w \in \mathcal{W}(x, u)\} .
$$

If

$$
\Phi:=f^{-1}(\Omega):=\{(x, u, w) \mid f(x, u, w) \in \Omega\},
$$

then the set of states that are robustly controllable to $\Omega$ is given by

$$
\operatorname{Pre}(\Omega)=\operatorname{Proj}_{X}(\Sigma),
$$

where

$$
\Sigma=\operatorname{Proj}_{X \times U}(\Pi) \backslash \operatorname{Proj}_{X \times U}(\Pi \backslash \Phi) .
$$

Proof. A graphical interpretation of the proof is given in Figure 1, where the set $\mathcal{W}\left(x_{a}, u_{a}\right)$ for a point $\left(x_{a}, u_{a}\right) \in \mathcal{Y}$ is also shown.

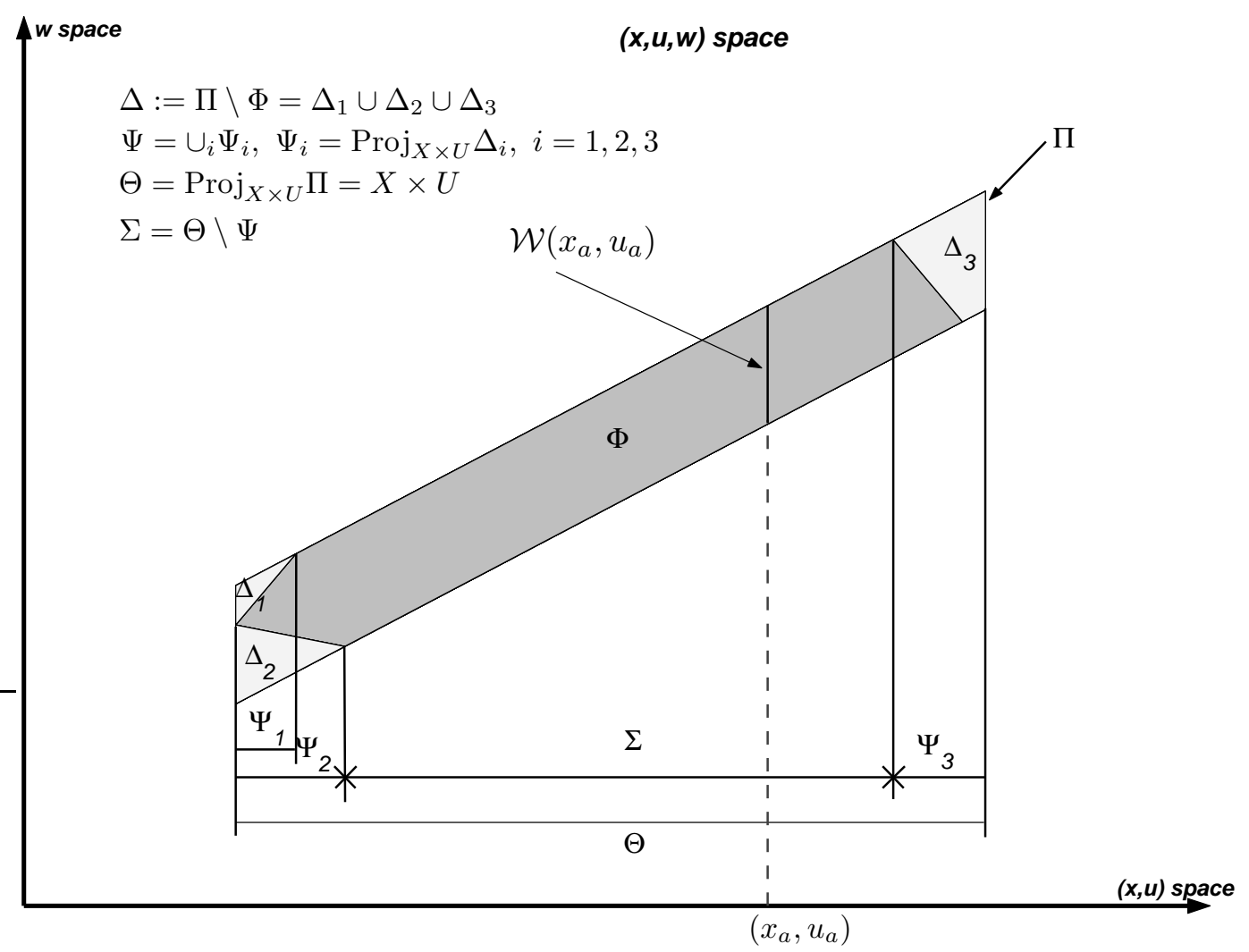

Figure 1: Graphical illustration of Theorem 1 
From the definition of the set difference,

$$
\Pi \backslash \Phi=\{(x, u, w) \mid(x, u) \in \mathcal{Y}, w \in \mathcal{W}(x, u) \text { and } f(x, u, w) \notin \Omega\}
$$

so that

$$
\operatorname{Proj}_{X \times U}(\Pi \backslash \Phi)=\{(x, u) \in \mathcal{Y} \mid \exists w \in \mathcal{W}(x, u) \text { such that } f(x, u, w) \notin \Omega\}
$$

and

$$
\mathcal{Y} \backslash \operatorname{Proj}_{X \times U}(\Pi \backslash \Phi)=\{(x, u) \in \mathcal{Y} \mid f(x, u, w) \in \Omega \text { for all } w \in \mathcal{W}(x, u)\} .
$$

It follows from $\mathbf{A} \mathbf{1}$ and (13) that

$$
\operatorname{Proj}_{X \times U}(\Pi)=\mathcal{Y}
$$

Hence

$$
\begin{aligned}
\operatorname{Proj}_{X \times U}(\Pi) \backslash \operatorname{Proj}_{X \times U}(\Pi \backslash \Phi) & =\mathcal{Y} \backslash \operatorname{Proj}_{X \times U}(\Pi \backslash \Phi) \\
& =\{(x, u) \in \mathcal{Y} \mid f(x, u, w) \in \Omega \text { for all } w \in \mathcal{W}(x, u)\} \\
& =\Sigma
\end{aligned}
$$

so that (16) is true.

The proof is completed by noting that

$$
\begin{aligned}
\operatorname{Proj}_{X}(\Sigma) & =\{x \mid \exists u \text { such that }(x, u) \in \mathcal{Y} \text { and } f(x, u, w) \in \Omega \text { for all } w \in \mathcal{W}(x, u)\} \\
& =\{x \mid \exists u \in \mathcal{U}(x) \text { such that } f(x, u, w) \in \Omega \text { for all } w \in \mathcal{W}(x, u)\} \\
& =\operatorname{Pre}(\Omega) .
\end{aligned}
$$

Remark 2. Note that the set $\Sigma$ defined in (12) is equal to $\operatorname{Proj}_{X \times U}(\Pi) \backslash \operatorname{Proj}_{X \times U}(\Pi \backslash \Phi)$, as stated in (16).

Theorem 2. Suppose $f: \mathbf{R}^{n} \times \mathbf{R}^{m} \times \mathbf{R}^{p} \rightarrow \mathbf{R}^{n}$ is continuous, $\mathcal{W}: \mathbf{R}^{r} \rightarrow 2^{\mathbf{R}^{p}}, r:=n+m$, is continuous and bounded on bounded sets. If $\Omega$ is closed, then $\operatorname{Pre}(\Omega)$ is closed.

Proof. Let the set-valued map $F: \mathbf{R}^{r} \rightarrow 2^{\mathbf{R}^{n}}$ be defined as follows:

$$
F(z):=\{f(z, w) \mid w \in \mathcal{W}(z)\}, \quad z:=(x, u) .
$$

By Proposition 1 in Appendix A, the set-valued function $F$ is continuous. The set $\Sigma$, defined in (16), is given by

$$
\Sigma=\{z \mid F(z) \subseteq \Omega\}=F^{\dagger}(\Omega) .
$$

Since $F$ is continuous and $\Omega$ is closed, it follows from Proposition 2 in Appendix A that $\Sigma$ is closed. Since $\operatorname{Pre}(\Omega)=\operatorname{Proj}_{X} \Sigma$, it follows that $\operatorname{Pre}(\Omega)$ is closed. 


\subsection{Special Cases}

Consider first the simpler case when the disturbance constraint set is a function of $x$ only, i.e. the disturbance $w$ satisfies $w \in \mathcal{W}(x)$. The definitions of $\Sigma$ and $\Pi$ in (12) and (13), respectively, and $\operatorname{Pre}(\Omega)$ become

$$
\begin{gathered}
\Sigma:=\{(x, u) \in \mathcal{Y} \mid f(x, u, w) \in \Omega \text { for all } w \in \mathcal{W}(x)\}, \\
\Pi:=\{(x, u, w) \mid(x, u) \in \mathcal{Y} \text { and } w \in \mathcal{W}(x)\}
\end{gathered}
$$

and

$$
\operatorname{Pre}(\Omega):=\{x \mid \exists u \in \mathcal{U}(x) \text { such that } f(x, u, w) \in \Omega \text { for all } w \in \mathcal{W}(x)\} .
$$

Theorem 1 remains true with these changes. A similar modification is needed if the disturbance constraint set is a function of $u$ only, i.e. the disturbance $w$ satisfies $w \in \mathcal{W}(u)$.

Remark 3. If the disturbance is independent of the state and input, Theorem 1 provides a method for computing the one-step robustly controllable set and is an alternative to the method in [10-12], where it is proposed to compute the so-called Pontryagin difference. Obviously, both methods will result in the same set. The difference between the two methods is that Theorem 1 relies on projection whereas the method in [10-12] does not. It is not easy to determine a priori which method would be more efficient. The computational requirements depend very much on the specifics of the problem and the computational tools that are available.

Next, consider the case when $f$ is a function of $(x, w)$ only, i.e. the system has no input $u$ and $x^{+}=f(x, w)$. In this case, the constraint $(x, u) \in \mathcal{Y}$ is replaced by $x \in \mathcal{X} \subset X$ and assumption $\mathbf{A} \mathbf{1}$ is replaced by:

A1': For all $x \in \mathcal{X}, \mathcal{W}(x) \neq \emptyset$ and $\mathcal{W}(\cdot)$ is bounded on bounded set.

Also, in this case the definitions of $\Sigma, \Pi$ and $\Phi$ in Theorem 1, and $\operatorname{Pre}(\Omega)$ are replaced by

$$
\begin{gathered}
\Sigma:=\{x \in \mathcal{X} \mid f(x, w) \in \Omega \text { for all } w \in \mathcal{W}(x)\}, \\
\Pi:=\{(x, w) \mid x \in \mathcal{X} \text { and } w \in \mathcal{W}(x)\}, \\
\Phi:=f^{-1}(\Omega):=\{(x, w) \mid f(x, w) \in \Omega\},
\end{gathered}
$$

and

$$
\operatorname{Pre}(\Omega):=\{x \in \mathcal{X} \mid f(x, w) \in \Omega \text { for all } w \in \mathcal{W}(x)\} .
$$

In other words, $\operatorname{Pre}(\Omega)$ is now the set of admissible states such that the successor state lies in $\Omega$ for all $w \in \mathcal{W}(x)$. In this case, the conclusion of Theorem 1 becomes

$$
\operatorname{Pre}(\Omega)=\Sigma=\operatorname{Proj}_{X}(\Pi) \backslash \operatorname{Proj}_{X}(\Pi \backslash \Phi) .
$$

As can be seen, this special case results in less computational effort, since operations are performed in lower-dimensional spaces and only two projection operations are needed. 


\subsection{Linear and Piecewise Affine $f(\cdot)$ with Additive State Disturbances}

Consider the system defined in (5) with

$$
f(x, u, w):=A_{q} x+B_{q} u+E_{q} w+c_{q} \text { if }(x, u, w) \in P_{q} .
$$

The sets $\left\{P_{q} \mid q \in Q\right\}$, where $Q$ has finite cardinality, are polyhedra and constitute a polyhedral partition of $\Pi$, i.e. $\Pi:=\bigcup_{q \in Q} P_{q}$ and the sets $P_{q}$ have non-intersecting interiors. For all $q \in Q$, the matrices $A_{q} \in \mathbf{R}^{n \times n}, B_{q} \in \mathbf{R}^{n \times m}, E_{q} \in \mathbf{R}^{n \times p}$ and vector $c_{q} \in \mathbf{R}^{n}$.

Theorem 3 (Piecewise affine systems). If the system is given by (32) and $\Pi$ and $\Omega$ are the unions of finite sets of polyhedra, then the robustly controllable set $\operatorname{Pre}(\Omega)$, as given in $(10)$ and (15), is the union of a finite set of polyhedra.

Proof. Let

$$
\Omega:=\bigcup_{j \in J} \Omega_{j},
$$

where $\left\{\Omega_{j} \mid j \in J\right\}$ is a finite set of polyhedra. First, note that

$$
\begin{aligned}
\Phi & =\bigcup_{j \in J}\left\{(x, u, w) \mid f(x, u, w) \in \Omega_{j}\right\} \\
& =\bigcup_{(j, q) \in J \times Q}\left\{(x, u, w) \in P_{q} \mid A_{q} x+B_{q} u+E_{q} w+c_{q} \in \Omega_{j}\right\} .
\end{aligned}
$$

Since $\left\{(x, u, w) \in P_{q} \mid A_{q} x+B_{q} u+E_{q} w+c_{q} \in \Omega_{j}\right\}$ is a polyhedron, it follows that $\Phi$ is the union of a finite set of polyhedra (i.e. a polygon).

As shown in Appendix B, the set difference between two polygons is a polygon. The proof is completed by recalling that the projection of the union of a finite number of sets is the union of the projections of the individual sets, hence the projection of a polygon is a polygon.

Remark 4. Clearly, Theorem 3 holds if the system is linear or affine (i.e. $Q$ has cardinality 1). It is interesting to observe that, even if $\Omega$ and $\Pi$ are both convex sets and $f(\cdot)$ is linear, there is no guarantee that $\operatorname{Pre}(\Omega)$ is convex. This is demonstrated in Section 4.1 via a numerical example.

Remark 5. See Appendix B for new results that allow one to compute the set difference between two (possibly non-convex) polygons. The projection of the set difference is then equal to the union of the projections of the individual polyhedra that constitute the set difference. The projection of each individual polyhedron can be computed, for example, via Fourier-Motzkin elimination [9] or via enumeration and projection of its vertices, followed by a convex hull computation [16]; see also $[6,7]$ for alternative projection methods.

\section{The $i$-step Set and Robustly Controlled Invariant Sets}

Consider the general case (Section 2.1). For any integer $i$, let $X_{i}$ denote the $i$-step (robustly controllable) set to $\Omega$, i.e. $X_{i}$ is the set of states that can be steered, by a time-varying state feedback control law, to the target set $\Omega$ in $i$ steps, for all allowable disturbance sequences 
while satisfying, at all times, the constraint $(x, u) \in \mathcal{Y}$. As is well-known $[3,11-13,17]$, the sequence of sets $\left\{X_{i}\right\}_{i=0}^{\infty}$ may be calculated recursively as follows:

$$
\begin{aligned}
X_{i+1} & =\operatorname{Pre}\left(X_{i}\right), \\
X_{0} & =\Omega .
\end{aligned}
$$

Before giving the next result, recall that a set $\mathcal{S}$ is robustly controlled invariant if and only if for any $x \in \mathcal{S}$, there exists a $u \in \mathcal{U}(x)$ such that $f(x, u, w) \in \mathcal{S}$ for all $w \in \mathcal{W}(x, u)$, i.e. $\mathcal{S}$ is robustly controlled invariant if and only if $\mathcal{S} \subseteq \operatorname{Pre}(\mathcal{S})[5,10]$. Recall also that the maximal robustly controlled invariant set $C_{\infty}$ in $\mathcal{X}$ is equal to the union of all robustly controlled invariant sets contained in $\mathcal{X}$.

Theorem 4. Suppose A1 holds:

(i) If the system is piecewise affine (defined by (32)) and if the sets $\Omega$ and $\Pi$ are the unions of finite sets of polyhedra, then each $i$-step set $X_{i}, i \in\{0,1, \ldots\}$, is the union of a finite set of polyhedra.

(ii) If $X_{j} \subseteq X_{j+1}$ for some $j \in\{0,1, \ldots\}$, then each set $X_{i}, i \in\{j, j+1, \ldots\}$, is robustly controlled invariant.

(iii) If the set $\Omega$ is robustly controlled invariant, then each set $X_{i}, i \in\{0,1, \ldots\}$, is robustly controlled invariant.

(iv) If $\Omega:=\mathcal{X}$ and $X_{j}=X_{j+1}$ for some $j \in\{0,1, \ldots\}$, then each set $X_{i}, i \in\{j, j+1, \ldots\}$, is equal to the maximal robustly controlled invariant set $C_{\infty}$ contained in $\mathcal{X}$.

Proof. The method of proof is standard and the reader is therefore referred to $[5,10]$.

Remark 6. Note that, if $\Omega \neq \mathcal{X}$ and $\Omega$ is robustly controlled invariant, then the maximal robustly controllable set $X_{\infty}$ to $\Omega\left(X_{\infty}=\bigcup_{i=0}^{\infty} X_{i}\right.$, where $\left.X_{0}=\Omega\right)$ is, in general, not equal to the maximal robustly controlled invariant set $C_{\infty}$ in $\mathcal{X}\left(C_{\infty}=\bigcap_{i=0}^{\infty} X_{i}\right.$, where $\left.X_{0}=\mathcal{X}\right)$.

Remark 7. As in Section 2.2, if the system has no input $u$, i.e. if $f$ is a function only of $(x, w)$, then with the appropriate modifications to definitions, Theorem 4 still holds, but with 'robustly controlled invariant' replaced with 'robustly positively invariant'.

\section{Numerical Examples}

In order to illustrate our results we consider two simple examples. In the first, the system is scalar and the disturbance state-dependent $(w \in \mathcal{W}(x))$; in the second, the system is second-order and the disturbance control-dependent $(w \in \mathcal{W}(u))$.

\subsection{Scalar System with State-dependent Disturbances}

We consider the following scalar system:

$$
x^{+}=x+u+w
$$




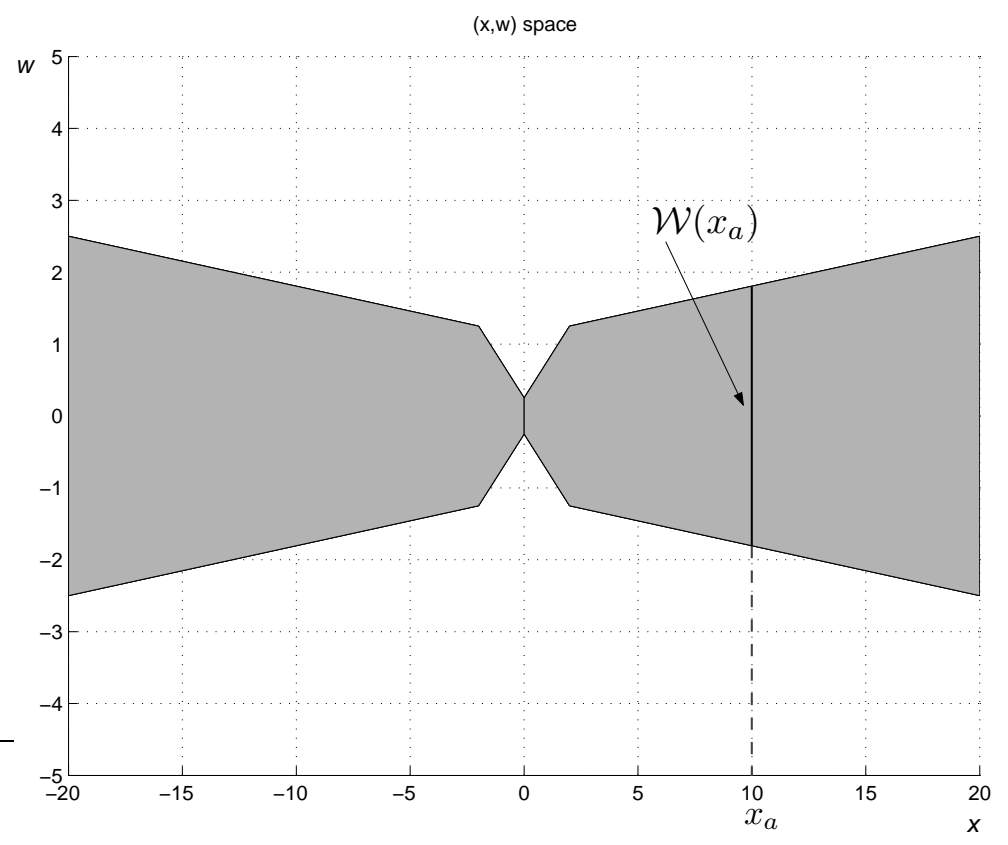

Figure 2: Graph of $\mathcal{W}$

which is subject to the constraints

$$
(x, u) \in \mathcal{X} \times \mathcal{U}, \mathcal{X}:=\{x \mid-5 \leq x \leq 20\} \text { and } \mathcal{U}:=\{u \mid-2 \leq u \leq 2\} .
$$

The state-dependent disturbance satisfies:

$$
w \in \mathcal{W}(x) \Leftrightarrow(x, w) \in \Delta:=\Delta_{1} \cup \Delta_{2},
$$

where $\Delta_{1}=$ convh $\{(0,0.25),(0,-0.25),(2,1.25),(2,-1.25),(20,2.25),(20,-2.25)\}$ and $\Delta_{2}=$ convh $\{(0,0.25),(0,-0.25),(-2,1.25),(-2,-1.25),(-20,2.25),(-20,-2.25)\}$. The set $\Delta$ is shown in Figure 2. The robustly controlled invariant target set is $X_{0}=\Omega=\{x \mid-0.6 \leq$ $x \leq 0.6\}$.

The sequence of $i$-step sets is computed by using the results of Theorem 1 and some of the sets are: $X_{1}=\{x \mid-0.7 \leq x \leq 0.7\}, X_{2}=\{x \mid-0.9 \leq x \leq 0.9\}, X_{3}=\{x \mid-1.3 \leq$ $x \leq 1.3\}, X_{4}=\{x \mid-2.0468 \leq x \leq 2.0468\}, \ldots, X_{8}=\{x \mid-4.5793 \leq x \leq 4.5793\}$, $X_{9}=\{x \mid-5 \leq x \leq 5.1131\}, X_{10}=\{x \mid-5 \leq x \leq 5.6123\}, \ldots, X_{49}=\{x \mid-5 \leq x \leq 12.2759\}$, $X_{50}=\{x \mid-5 \leq x \leq 12.3099\}$. The set $X_{\infty}$ of all states that can be steered to the target set, while satisfying state and control constraints, for all allowable disturbance sequences, is: $X_{\infty}=\{x \mid-5 \leq x \leq 12.7999\}$. The sets $\Sigma_{i}$ for $i=1,2,3,4$ are also shown in Figure 3.

In order to illustrate the fact that the $i$-step sets can be non-convex even if $\mathcal{X}, \mathcal{U}, \Omega$ and the graph of $\mathcal{W}(x)$ are convex, consider the same example. This time the state-dependent disturbance satisfies:

$$
w \in \mathcal{W}(x) \Leftrightarrow(x, w) \in \Delta:=\operatorname{convh}\{(-5,0),(0,-3),(5,0),(0,3)\} .
$$

If the target set is $X_{0}=\Omega=\{x \mid-2.5 \leq x \leq 2.5\}$, the one-step set is $X_{1}=\{x \mid-3.75 \leq x \leq$ $-0.8333\} \cup\{x \mid 0.8333 \leq x \leq 3.75\}$. The sets $\Delta$ and $\Sigma$ are shown in Figure 4. 


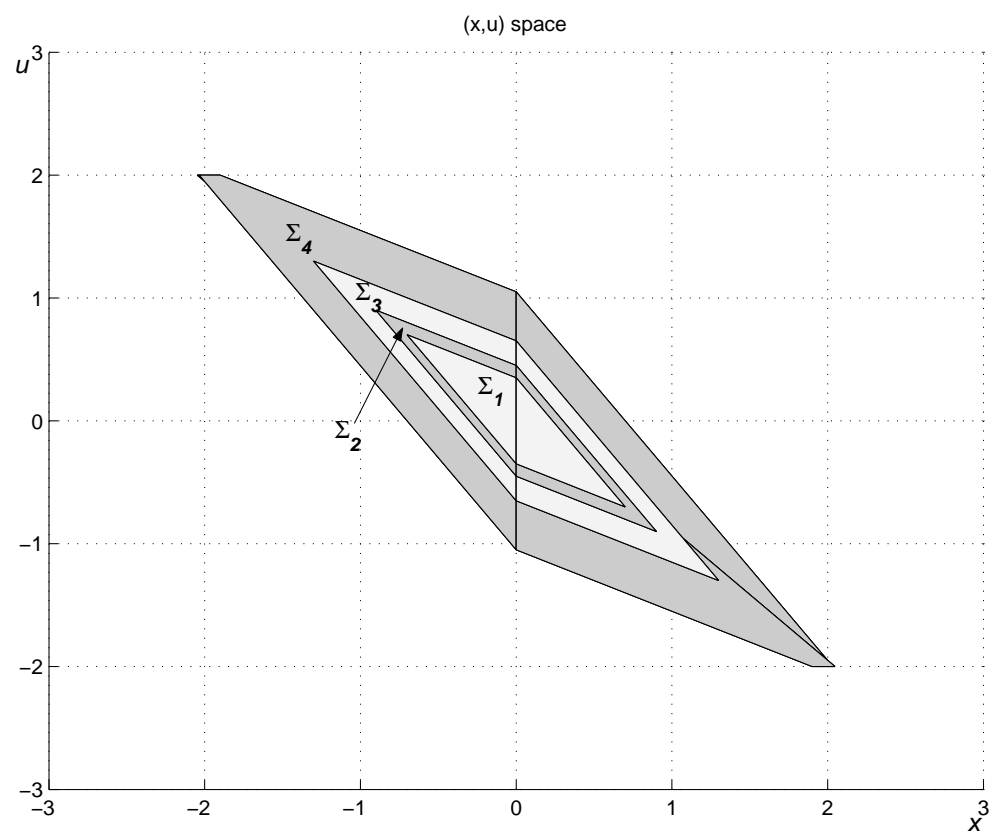

Figure 3: Sets $\Sigma_{i}$ for $i=1,2,3,4$
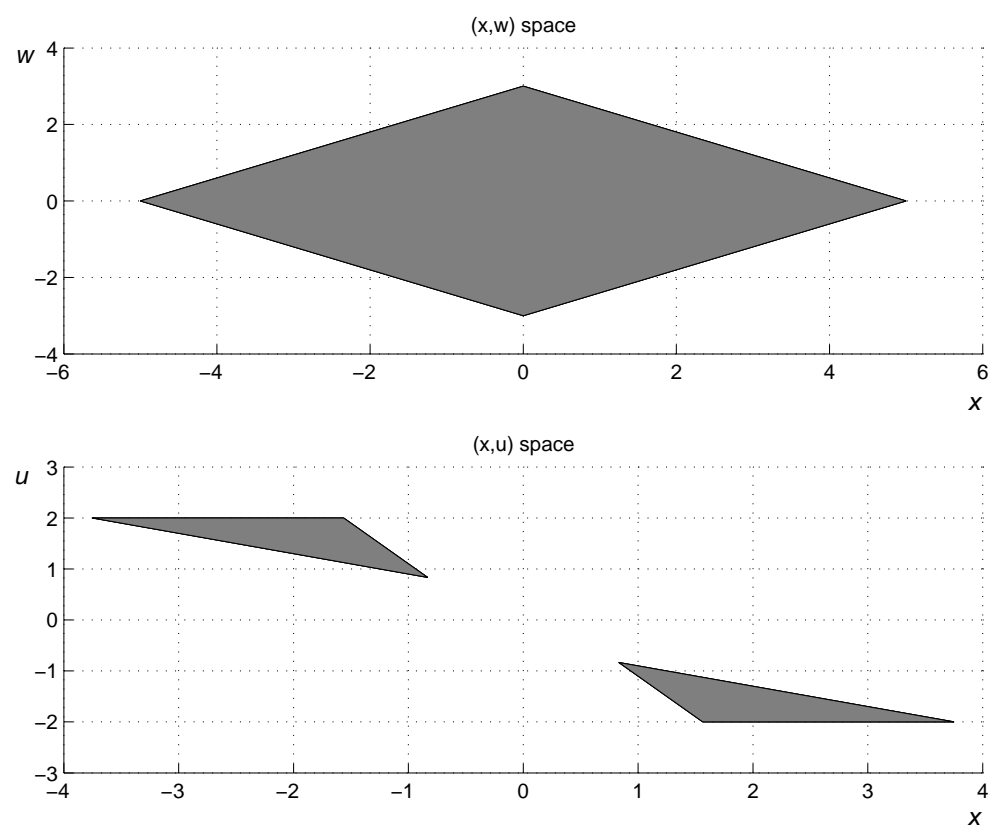

Figure 4: Graph of $\mathcal{W}$ (top) and the set $\Sigma$ (bottom) 

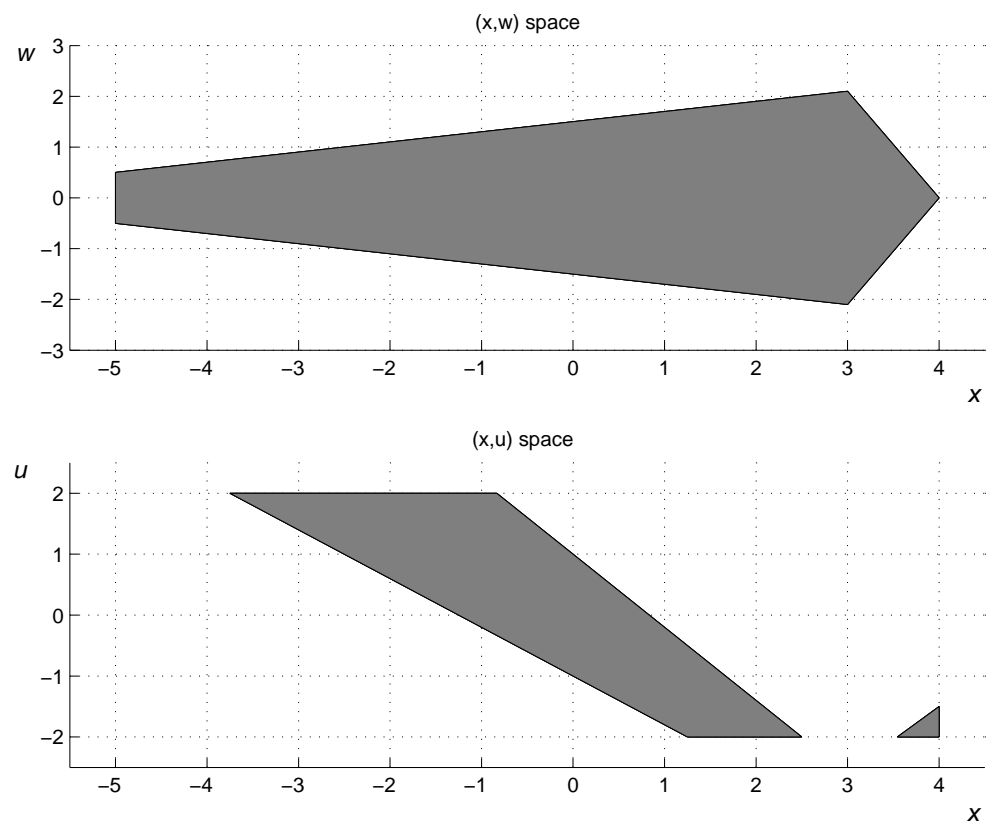

Figure 5: Graph of $\mathcal{W}$ (top) and the set $\Sigma$ (bottom)

Even if $\Omega$ is a robustly controlled invariant set, the convexity of each $i$-step set still cannot be guaranteed. This is easily illustrated by considering the same example with $\mathcal{X}=\{x \mid-5 \leq$ $x \leq 4\}, w \in \mathcal{W}(x) \Leftrightarrow(x, w) \in \Delta:=\operatorname{convh}\{(-5,0.5),(-5,-0.5),(3,-2.1),(4,0),(3,2.1)\}$ and the robustly controlled invariant target set $X_{0}=\Omega=\{x \mid-2.5 \leq x \leq 2.5\}$. In this case, the one-step robustly controlled invariant set is $X_{1}=\{x \mid-3.75 \leq x \leq 2.5\} \cup\{x \mid 3.5455 \leq x \leq$ $4\}$. The sets $\Delta$ and $\Sigma$ are shown in Figure 5 .

\subsection{Second-order LTI Example with Control-dependent Disturbances}

The discrete-time linear time-invariant system

$$
x^{+}=\left[\begin{array}{cc}
0.7969 & -0.2247 \\
0.1798 & 0.9767
\end{array}\right] x+\left[\begin{array}{l}
0.1271 \\
0.0132
\end{array}\right] u+w
$$

is subject to the state and control constraints

$$
(x, u) \in \mathcal{X} \times \mathcal{U}, \mathcal{X}:=\left\{x \mid\|x\|_{\infty} \leq 10,[-11] x \leq 12\right\}, \mathcal{U}:=\{u \mid-3 \leq u \leq 3\} .
$$

The control-dependent disturbance satisfies:

$$
w \in \mathcal{W}(u) \Leftrightarrow(u, w) \in \Delta:=\Delta_{1} \cup \Delta_{2},
$$

where $\Delta_{1}$ and $\Delta_{2}$ are given by:

$$
\Delta_{1}=\left\{(u, w) \mid\left[\begin{array}{ccc}
-0.008 & 0 & -1 \\
-1 & 0 & 0 \\
-0.008 & 1 & 0 \\
-0.008 & -1 & 0 \\
-0.008 & 0 & 1
\end{array}\right]\left[\begin{array}{c}
u \\
w
\end{array}\right] \leq\left[\begin{array}{c}
0.01 \\
0 \\
0.01 \\
0.01 \\
0.01
\end{array}\right]\right\},
$$



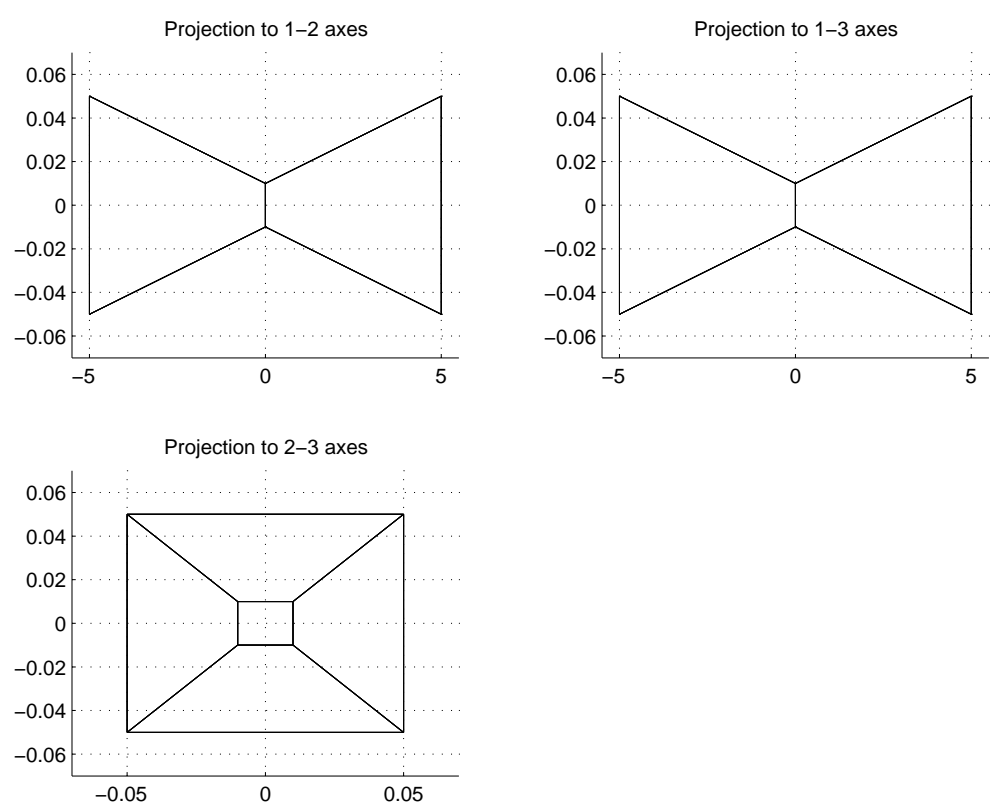

Figure 6: Graph of $\mathcal{W}$

and

$$
\Delta_{2}=\left\{(u, w) \mid\left[\begin{array}{ccc}
0.008 & 0 & 1 \\
10 & 0 & \\
0.008 & -1 & 0 \\
0.008 & 1 & 0 \\
0.008 & 0 & -1
\end{array}\right]\left[\begin{array}{c}
u \\
w
\end{array}\right] \leq\left[\begin{array}{c}
0.01 \\
0 \\
0.01 \\
0.01 \\
0.01
\end{array}\right]\right\}
$$

The robustly controlled invariant target set is $X_{0}=\operatorname{convh}\{(-0.2035,0.0482)$, $(0.2035,-0.0482),(-0.2035,-0.0148),(-0.1405,0.0482),(0.2035,0.0148),(0.1405,-0.0482)\}$. The projections of the set $\Delta$ onto two-dimensional subspaces are shown in Figure 6 . Some of the $i$-step sets, computed using Theorem 1, are shown in Figure 7.

\section{Conclusions}

The main result of this paper (Theorem 1 ) showed how one can obtain $\operatorname{Pre}(\Omega)$, the set of states that can be robustly steered to $\Omega$, via the computation of a sequence of set differences and projections. It was then shown in Theorem 3 that if $\Omega$ and the relevant constraint sets are polygons (i.e. they are given by the unions of finite sets of convex polyhedra) and the system is linear or piecewise affine, then $\operatorname{Pre}(\Omega)$ is also a polygon and can be computed using standard computational geometry software. In particular, new results were given in Appendix B which allow one to compute the set difference for (possibly non-convex) polygons by solving a finite number of LPs. It was then shown in Section 3 how Pre $(\cdot)$ can be used to recursively compute the $i$-step set, i.e. the set of states which can be robustly steered to a given target set in $i$ steps, as well as how $\operatorname{Pre}(\cdot)$ can be used to compute the maximal robustly controlled invariant set. Finally, some simple examples were given which show that, even if the system is linear, 


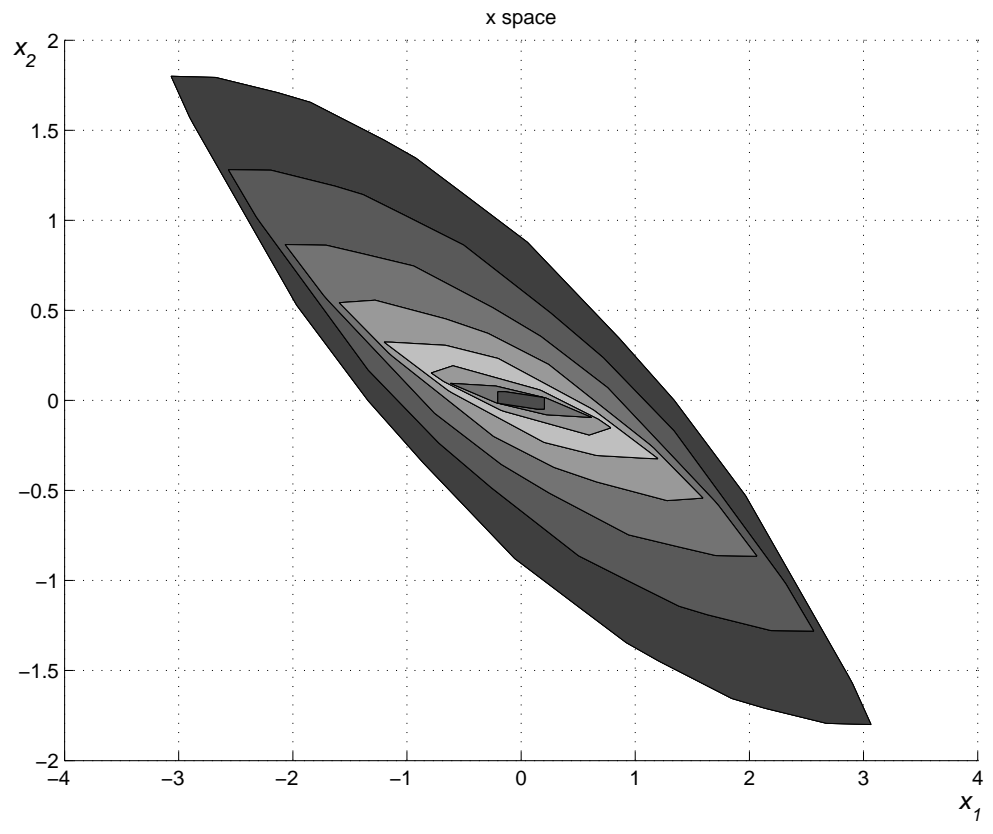

Figure 7: Sets $X_{i}$ for $i=0,1, \ldots, 7$

the respective constraint sets are convex and the target set is robustly controlled invariant, convexity of the $i$-step sets cannot be guaranteed.

\section{Acknowledgments}

This research was supported by the Engineering and Physical Sciences Research Council and the Royal Academy of Engineering, United Kingdom. The authors are grateful to Professor E. Polak for helpful discussions. All computations were performed with the Geometric Bounding Toolbox [16].

\section{Appendices}

\section{A Results on Set-valued Functions}

The definitions of inner and outer semi-continuity employed below are due to Rockafellar and Wets [15]; for Definitions 1-4 and Theorem 5, see [14]; Polak also provided the proof of Proposition 1 (private communication). In what follows, $B(z, \rho):=\{z \mid\|z\| \leq \rho\}$ and $d(a, A):=\inf _{b \in A}\|a-b\|$.

Definition 1. A set-valued map $F: \mathbf{R}^{r} \rightarrow 2^{\mathbf{R}^{n}}$ is outer semi-continuous (o.s.c.) at $\hat{z}$ if $F(\hat{z})$ is closed and, for every compact set $S$ such that $F(\hat{z}) \cap S=\emptyset$, there exists a $\rho>0$ such that $F(z) \cap S=\emptyset$ for all $z \in B(\hat{z}, \rho)$. A set-valued map $F: \mathbf{R}^{r} \rightarrow 2^{\mathbf{R}^{n}}$ is o.s.c. if it is o.s.c. at every $z \in \mathbf{R}^{r}$. 
Definition 2. A set-valued map $F: \mathbf{R}^{r} \rightarrow 2^{\mathbf{R}^{n}}$ is inner semi-continuous (i.s.c.) at $\hat{z}$ if $F(\hat{z})$ is closed and, for every open set $S$ such that $F(\hat{z}) \cap S \neq \emptyset$, there exists a $\rho>0$ such that $F(z) \cap S \neq \emptyset$ for all $z \in B(\hat{z}, \rho)$. A set-valued map $F: \mathbf{R}^{r} \rightarrow 2^{\mathbf{R}^{n}}$ is i.s.c. if it is i.s.c. at every $z \in \mathbf{R}^{r}$.

Definition 3. A set-valued map $F: \mathbf{R}^{r} \rightarrow 2^{\mathbf{R}^{n}}$ is continuous if it is both o.s.c. and i.s.c.

Definition 4. A point $\hat{a}$ is a limit point of the infinite sequence of sets $\left\{A_{i}\right\}$ if $d\left(\hat{a}, A_{i}\right) \rightarrow 0$. A point $\hat{a}$ is a cluster point if there exists a subsequence $I \subset N$ such that $d\left(\hat{a}, A_{i}\right) \rightarrow 0$ as $i \rightarrow \infty$, $i \in I$. The set $\lim \sup A_{i}$ is the set of cluster points of $\left\{A_{i}\right\}$ and $\lim \inf A_{i}$ is the set of limit points of $\left\{A_{i}\right\}$, i.e. $\limsup A_{i}$ is the set of cluster points of sequences $\left\{a_{i}\right\}$ such that $a_{i} \in A_{i}$ for all $i \in \mathbf{N}$ and $\lim \inf A_{i}$ is the set of limits of sequences $\left\{a_{i}\right\}$ such that $a_{i} \in A_{i}$ for all $i \in \mathbf{N}$. The sets $A_{i}$ converge to the set $A\left(A_{i} \rightarrow A\right.$ or $\left.\lim A_{i}=A\right)$ if $\lim \sup A_{i}=\liminf A_{i}=A$.

The following result appears as Theorem 5.3.7 in [14].

Theorem 5. (i) A function $F: \mathbf{R}^{r} \rightarrow 2^{\mathbf{R}^{n}}$ is o.s.c. at $\hat{z}$ if and only if for any sequence $\left\{z_{i}\right\}$ such that $z_{i} \rightarrow \hat{z}, \lim \sup F\left(z_{i}\right) \subseteq F(\hat{z})$. Also, $F$ is o.s.c. if and only if it graph $G:=\{(z, y) \mid$ $y \in F(z)\}$ is closed.

(ii) A function $F: \mathbf{R}^{r} \rightarrow 2^{\mathbf{R}^{n}}$ is i.s.c. at $\hat{z}$ if and only if for any sequence $\left\{z_{i}\right\}$ such that $z_{i} \rightarrow \hat{z}, \liminf F\left(z_{i}\right) \supseteq F(\hat{z})$.

(iii) Suppose $F: \mathbf{R}^{r} \rightarrow 2^{\mathbf{R}^{n}}$ is such that $F(z)$ is compact for all $z \in \mathbf{R}^{r}$ and bounded on bounded sets. Then $F$ is o.s.c. at $\hat{z}$ if and only if, for every open set $S$ such that $F(\hat{z}) \subseteq S$, there exists a $\rho>0$ such that $F(z) \subseteq S$ for all $z \in B(\hat{z}, \rho)$.

Proposition 1. Suppose that $f: \mathbf{R}^{r} \times \mathbf{R}^{p} \rightarrow \mathbf{R}^{n}$ is continuous and that $\mathcal{W}: \mathbf{R}^{p} \rightarrow 2^{\mathbf{R}^{p}}$ is continuous and bounded on bounded sets. Then the set-valued function $F: \mathbf{R}^{r} \rightarrow 2^{\mathbf{R}^{n}}$ defined by $F(z):=\{f(z, w) \mid w \in \mathcal{W}(z)\}$ is continuous.

Proof. (i) ( $F$ is o.s.c.). Let $\left\{z_{i}\right\}$ be any infinite sequence such that $z_{i} \rightarrow \hat{z}$ and let $\left\{f_{i}\right\}$ be any infinite sequence such that $f_{i} \in F\left(z_{i}\right)$ for all $i \in \mathbf{N}$ and $f_{i} \rightarrow \hat{f}$. Then, for all $i, f_{i}=f\left(z_{i}, w_{i}\right)$ with $w_{i} \in \mathcal{W}\left(z_{i}\right)$. Since $\left\{z_{i}\right\}$ lies in a compact set and $\mathcal{W}: \mathbf{R}^{p} \rightarrow 2^{\mathbf{R}^{p}}$ is bounded on bounded sets, there exists a subsequence of $\left\{w_{i}\right\}$ such that $w_{i} \rightarrow \hat{w}$ as $i \rightarrow \infty, i \in I \subset \mathbf{N}$. Since $\mathcal{W}$ is continuous, $\hat{w} \in \mathcal{W}(\hat{z})$. Hence

$$
\hat{f}=\lim _{i \in I} f\left(z_{i}, w_{i}\right)=f(\hat{z}, \hat{w}) \in F(\hat{z}) .
$$

This implies that $F$ is o.s.c.

(ii) ( $F$ is i.s.c.) Let $\left\{z_{i}\right\}$ be any infinite sequence such that $z_{i} \rightarrow \hat{z}$ and let $\hat{f}$ be an arbitrary point in $F(\hat{z})$. Then $\hat{f}=F(\hat{z}, \hat{w})$ for some $\hat{w} \in F(\hat{z})$. Since $\mathcal{W}$ is continuous, there exists an infinite sequence $\left\{w_{i}\right\}$ such that $w_{i} \in \mathcal{W}\left(z_{i}\right)$ and $w_{i} \rightarrow \hat{w}$. Then $f_{i}:=f\left(z_{i}, w_{i}\right) \in F\left(z_{i}\right)$ for all $i \in \mathbf{N}$ and

$$
\lim f_{i}=\lim f\left(z_{i}, w_{i}\right)=f(\hat{z}, \hat{w})=\hat{f} \in F(\hat{z})
$$

This implies that $F$ is i.s.c.

Proposition 2. Suppose $F: \mathbf{R}^{r} \rightarrow 2^{\mathbf{R}^{n}}$ is continuous and that $\Omega \subseteq \mathbf{R}^{n}$ is closed. Then the (outer) inverse set $F^{\dagger}(\Omega):=\{z \mid F(z) \subseteq \Omega\}$ is closed. 
Proof. Suppose $\left\{z_{i}\right\}$ is an arbitrary infinite sequence in $F^{\dagger}(\Omega)\left(F\left(z_{i}\right) \subseteq \Omega\right.$ for all $\left.i \in \mathbf{N}\right)$ such that $z_{i} \rightarrow \hat{z}$. Since $F$ is continuous, $\lim F\left(z_{i}\right)=F(\hat{z})$. Because $\Omega$ is closed, $F\left(z_{i}\right) \subseteq \Omega$ for all $i \in \mathbf{N}$ implies $F(\hat{z}) \subseteq \Omega$. Hence $\hat{z} \in F^{\dagger}(\Omega)$ so that $F^{\dagger}(\Omega)$ is closed.

\section{B Set Difference of Polygons}

In the following, $\mathcal{N}_{n}:=\{1,2, \ldots, n\}$.

The first result, which is adapted from [1, Thm. 3], allows one to compute the set difference of two polyhedra:

Proposition 3. Let $A \subset \mathbf{R}^{n}$ and $B:=\left\{x \in \mathbf{R}^{n} \mid c_{i}^{\prime} x \leq d_{i}, i=1, \ldots, r\right\}$ be non-empty polyhedra, where all the $c_{i} \in \mathbf{R}^{n}$ and $d_{i} \in \mathbf{R}$. If

$$
\begin{aligned}
& S_{1}:=\left\{x \in A \mid c_{1}^{\prime} x>d_{1}\right\}, \\
& S_{i}:=\left\{x \in A \mid c_{i}^{\prime} x>d_{i}, c_{j}^{\prime} x \leq d_{j}, \forall j \in \mathcal{N}_{i-1}\right\}, \quad i=2, \ldots, r,
\end{aligned}
$$

then $A \backslash B=\bigcup_{i=1}^{r} S_{i}$ is a polygon. Furthermore, $\left\{S_{i} \neq \emptyset \mid i \in \mathcal{N}_{r}\right\}$ is a partition of $A \backslash B$.

Proof. See the proof of [1, Thm. 3].

Remark 8. In practice, computation time can be reduced by checking whether $A \cap B$ is empty or whether $A \subseteq B$ before actually computing $A \backslash B$; if $A \cap B=\emptyset$, then $A \backslash B=A$ and if $A \subseteq B$, then $A \backslash B=\emptyset$. Using an extended version of Farkas' Lemma [5, Lem. 4.1], checking whether one polyhedron is contained in another amounts to solving a single LP. Alternatively, one can solve a finite number of smaller LPs to check for set inclusion [10, Prop. 3.4].

Remark 9. Once $A \backslash B$ has been computed, the memory requirements can be reduced by removing all empty $S_{i}$ and removing any redundant inequalities describing the non-empty $S_{i}$. Checking whether a polyhedron is non-empty can be done by solving a single linear program (LP). Removing redundant inequalities can be done by solving a finite number of LPs [10, App. B]. As a result, it is a good idea to determine first whether an $S_{i}$ is non-empty or not before removing redundant inequalities.

The second result allows one to compute the set difference of a polygon and a polyhedron:

Proposition 4. Let $C:=\bigcup_{j=1}^{p} C_{j}$ be a polygon, where all the $C_{j}, j \in \mathcal{N}_{p}$, are non-empty polyhedra. If $A$ is a non-empty polyhedron, then

$$
C \backslash A=\bigcup_{j=1}^{p}\left(C_{j} \backslash A\right)
$$

is a polygon.

Proof. This follows trivially from the fact that $C \backslash A=\left(\bigcup_{j=1}^{p} C_{j}\right) \cap A^{\mathrm{c}}=\bigcup_{j=1}^{p}\left(C_{j} \cap A^{\mathrm{c}}\right)$.

Remark 10. If $\left\{C_{j} \mid j \in \mathcal{N}_{p}\right\}$ is a partition of $C$ and $C \backslash A \neq \emptyset$, then $\left\{C_{j} \backslash A \neq \emptyset \mid j \in \mathcal{N}_{p}\right\}$ is a partition of $C \backslash A$ if Proposition 3 is used to compute each polygon $C_{j} \backslash A, j \in \mathcal{N}_{p}$. 
The last result allows one to compute the set difference of two polygons:

Proposition 5. Let $C:=\bigcup_{j=1}^{p} C_{j}$ and $D:=\bigcup_{k=1}^{q} D_{k}$ be polygons, where all the $C_{j}, j \in \mathcal{N}_{p}$, and $D_{k}, k \in \mathcal{N}_{q}$, are non-empty polyhedra. If

$$
\begin{aligned}
& E_{0}:=C, \\
& E_{k}:=E_{k-1} \backslash D_{k}, \quad \forall k \in \mathcal{N}_{q},
\end{aligned}
$$

then $C \backslash D=E_{q}$ is a polygon.

Proof. The result follows from noting that

$$
\begin{aligned}
C \backslash D & =C \cap D^{\mathrm{c}} \\
& =C \cap\left(\cup_{k=1}^{q} D_{k}\right)^{\mathrm{c}} \\
& =C \cap\left(\cap_{k=1}^{q} D_{k}^{\mathrm{c}}\right) \\
& =C \cap D_{1}^{\mathrm{c}} \cap D_{2}^{\mathrm{c}} \cap \cdots \cap D_{q}^{\mathrm{c}} \\
& =\left(C \cap D_{1}^{\mathrm{c}}\right) \cap D_{2}^{\mathrm{c}} \cap \cdots \cap D_{q}^{\mathrm{c}} \\
& =\left(C \backslash D_{1}\right) \cap D_{2}^{\mathrm{c}} \cap \cdots \cap D_{q}^{\mathrm{c}} \\
& =\left(\left(C \backslash D_{1}\right) \backslash D_{2}\right) \cap \cdots \cap D_{q}^{\mathrm{c}} \\
& =\left(\cdots\left(\left(C \backslash D_{1}\right) \backslash D_{2}\right) \backslash \cdots\right) \backslash D_{q}
\end{aligned}
$$

and letting $E_{0}:=C$ and $E_{k}:=E_{k-1} \backslash D_{k}, \forall k \in \mathcal{N}_{q}$, yields the claim.

Remark 11. Each polygon $E_{k-1} \backslash D_{k}, k \in \mathcal{N}_{q}$, can be computed using Proposition 4.

Remark 12. Note also that if $\left\{C_{j} \mid j \in \mathcal{N}_{p}\right\}$ is a partition of $C$ and $C \backslash D \neq \emptyset$, then the sets which define $E_{q}$ form a partition of $C \backslash D$ if Propositions 3 and 4 were used to compute all the $E_{k}, k \in \mathcal{N}_{q}$.

\section{References}

[1] A. Bemporad, M. Morari, V. Dua, and E.N. Pistikopoulos. The explicit linear quadratic regulator for constrained systems. Automatica, 38:3-20, 2002.

[2] D.P. Bertsekas. Infinite-time reachability of state-space regions by using feedback control. IEEE Trans. Automatic Control, AC-17(5):604-613, 1972.

[3] D.P. Bertsekas and I.B. Rhodes. On the minimax reachability of target sets and target tubes. Automatica, 7:233-247, 1971.

[4] F. Blanchini. Ultimate boundedness control for uncertain discrete-time systems via setinduced Lyapunov functions. IEEE Trans. Automatic Control, 39(2):428-433, 1994.

[5] F. Blanchini. Set invariance in control. Automatica, 35(11):1747-1767, 1999. Survey paper. 
[6] P. D'Alessandro. A conical approach to linear programming. Scalar and vector optimization problems. Gordon and Breach Science Publishers, 1997.

[7] P. D'Alessandro, M. Dalla Mora, and E. De Santis. Techniques of linear programming based on the theory of convex cones. Optimization, 20:761-777, 1989.

[8] E. De Santis. Invariant sets: A generalization to constrained systems with state dependent disturbances. In Proc. 37th IEEE Conference on Decision and Control, pages 622-3, Tampa, Florida, USA, December 1998.

[9] S.S. Keerthi and E.G. Gilbert. Computation of minimum-time feedback control laws for discrete-time systems with state-control constraints. IEEE Trans. Automatic Control, AC-32:432-435, 1987.

[10] E.C. Kerrigan. Robust Constraint Satisfaction: Invariant Sets and Predictive Control. PhD thesis, Department of Engineering, University of Cambridge, 2000. Downloadable from http://www-control.eng.cam.ac.uk/eck21/.

[11] E.C. Kerrigan, J. Lygeros, and J.M. Maciejowski. A geometric approach to reachability computations for constrained discrete-time systems. In Proc. 15th IFAC World Congress on Automatic Control, Barcelona, Spain, July 2002.

[12] E.C. Kerrigan and D.Q. Mayne. Optimal control of constrained, piecewise affine systems with bounded disturbances. In Proc. 41st IEEE Conference on Decision and Control, Las Vegas, Nevada, USA, December 2002.

[13] D.Q. Mayne. Control of constrained dynamic systems. European Journal of Control, 7:87-99, 2001. Survey paper.

[14] E. Polak. Optimization: Algorithms and Consistent Approximations. Springer-Verlag, New York, 1997. ISBN 0-387-94971-2.

[15] R.T. Rockafellar and R.J-B. Wets. Variational Analysis. Springer-Verlag, 1998.

[16] S.M. Veres. Geometric Bounding Toolbox (GBT 7.2) for MatlaB. Official website: http://sysbrain.com/gbt/.

[17] R. Vidal, S. Schaffert, O. Shakernia, J. Lygeros, and S. Sastry. Decidable and semidecidable controller synthesis for classes of discrete time hybrid systems. In Proc. 40th IEEE Conference on Decision and Control, Orlando, Florida, USA, December 2001. 